\title{
Stability Analysis of an SIR Epidemic Model with Non-Linear Incidence Rate and Treatment
}

\author{
Olukayode Adebimpe ${ }^{1 *}$, Kehinde Adekunle Bashiru'2, Taiwo Adetola Ojurongbe ${ }^{2}$ \\ ${ }^{1}$ Department of Industrial Mathematics, Landmark University, Omuaran, Nigeria \\ ${ }^{2}$ Department of Mathematical and Physical Science, Osun State University, Osogbo, Nigeria \\ Email: *lukayode1978@gmail.com
}

Received 7 May 2015; accepted 29 June 2015; published 2 July 2015

Copyright (C) 2015 by authors and Scientific Research Publishing Inc.

This work is licensed under the Creative Commons Attribution International License (CC BY).

http://creativecommons.org/licenses/by/4.0/

(c) (i) Open Access

\begin{abstract}
We consider a SIR epidemic model with saturated incidence rate and treatment. We show that if the basic reproduction number, $R_{0}$ is less than unity and the disease free equilibrium is locally asymptotically stable. Moreover, we show that if $R_{0}>1$, the endemic equilibrium is locally asymptotically stable. In the end, we give some numerical results to compare our model with existing model and to show the effect of the treatment term on the model.
\end{abstract}

\section{Keywords}

SIR Epidemic Model, Basic Reproduction Number, Local Stability, Treatment, Saturated Incidence Rate

\section{Introduction}

While mathematical modeling of infectious diseases could be traced back to 1760 when Bernoulli used mathematical models for small pox [1], the research in infectious diseases, using deterministic mathematical models actually began in $20^{\text {th }}$ century.

Bilinear and standard incidence rates have been frequently used in classical epidemic models [2]. Several different incidence rates have been proposed by researchers. After a study of the cholera epidemic spread in Bari in 1973, Capasso and Serio [3] introduced a saturated incidence rate g(I)S into epidemic models. Ruan and Wang [4] studied an epidemic model with specific nonlinear incidence rate of the form $\frac{K I^{l} S}{1+\alpha I^{h}}$ where $l$ and h were

${ }^{*}$ Corresponding author.

How to cite this paper: Adebimpe, O., Bashiru, K.A. and Ojurongbe, T.A. (2015) Stability Analysis of an SIR Epidemic Model with Non-Linear Incidence Rate and Treatment. Open Journal of Modelling and Simulation, 3, 104-110.

http://dx.doi.org/10.4236/ojmsi.2015.33011 
positive constants and obtained lots of interesting dynamical behaviour of the model such as a limit cycle, two limit cycles and homoclimic loop etc. In 2000, van den Driessche and Watmough [5] studied an SIS epidemic model with the incidence rate of the form $B S I\left(1+\alpha I^{k-1}\right)$ where $\beta>0, \alpha \geq 0$ and $k>0$. Xiao and Ruan [6] considered a special nonlinear incident rate of the form $\frac{K I S}{1+\alpha I^{2}}$ where $g(I)$ is non-monotone.

Jasmine and Amirtharaj [7] on account of the effect of limited treatment resources on the control of epidemic disease incorporated a modified SIR epidemic model with generalized incidence rate. They discussed the stability analysis of the disease-free equilibrium and endemic equilibrium with a nonlinear incidence rate. Chauhan et al. [8] discussed the stability analysis pf SIR epidemic model with and without vaccination. They discussed the local and global stability of the model through the basis reproduction number

In a recent paper, Kaddar [9] considered a delayed SIR epidemic model with a saturated incidence rate of the form $\frac{\beta S I}{1+\alpha_{1} S+\alpha_{2} I}$ and Pathak et al. [10] also considered the transmission rate $\phi=\frac{K I}{1+\alpha S+\beta I}$ which displayed a saturation effect accounting for the fact that the number of contacts in individual reaches some maximal value done to spatial or social distribution of the population.

In this paper, we extend the work of Pathak et al., by considering a transmission rate $\phi=\frac{K I}{1+\alpha S^{p}+\beta I^{q}}$ where $p=q>1$. We also look at the effect of the transmission rate on the SIR epidemic model and we include the treatment term $\tau I$ where $\tau I=r I, I>0$.

\section{The Basic Mathematical Model}

We modify the model of Pathak et al., by extending the transmission rate to nonlinear orders and also include the treatment term.

\section{Pathak et al. Model}

$$
\begin{aligned}
\frac{\mathrm{d} S}{\mathrm{~d} t} & =b-d S-\frac{K I}{1+\alpha S+\beta I}+\gamma R \\
\frac{\mathrm{d} I}{\mathrm{~d} t} & =\frac{K I}{1+\alpha S+\beta I}-(d+\mu) I \\
\frac{\mathrm{d} R}{\mathrm{~d} t} & =\mu I-(d+\gamma) R
\end{aligned}
$$

\section{Modified Model}

The modified model is as follows:

$$
\begin{aligned}
& \frac{\mathrm{d} S}{\mathrm{~d} t}=b-d S-\frac{K I}{1+\alpha S^{p}+\beta I^{q}}+\gamma R \\
& \frac{\mathrm{d} I}{\mathrm{~d} t}=\frac{K I}{1+\alpha S^{p}+\beta I^{q}}-(d+\mu) I-\tau I \\
& \frac{\mathrm{d} R}{\mathrm{~d} t}=\mu I-(d+\gamma) R+\tau I
\end{aligned}
$$

where the transmission rate $\phi=\frac{K I}{1+\alpha S^{p}+\beta I^{q}}$ and $\tau I=r I, I>0$.

where $S(t), I(t)$ and $R(t)$ represent the number of susceptible, infective and recovered individuals at time $t$, respectively, $b$ is the recruitment rate of the population, $d$ is the natural death rate of the population, $k$ is the proportionality constant, $\mu$ is the natural recovery rate of the infective individuals, $\gamma$ is the rate at which recovered individuals lose immunity and return to the susceptible class, $\alpha$ and $\beta$ are the parameters which measure the effects of sociological, psychological or other mechanisms and $p$ and $q$ are positive constants which are greater than unity. 


\section{Steady State and Local Stability of the Critical Points}

In this section, we discuss the local stability of the disease-free equilibrium and endemic equilibrium of system (2). The system (2) has a disease free of $R_{0}=\frac{\mu \frac{b}{d}}{\left(1+\alpha\left(\frac{b}{d}\right)^{p}\right)(d+\mu+r)}>1, E_{0}=\left(\frac{b}{e}, 0,0\right)$. Further, system ( admits a unique endemic equilibrium $E_{*}\left(S_{*}, I_{*}, R_{*}\right)$

Proposition 1: If $R_{0}<1$, then the disease free equilibrium $E_{0}$ is locally asymptotically stable.

\section{Proof:}

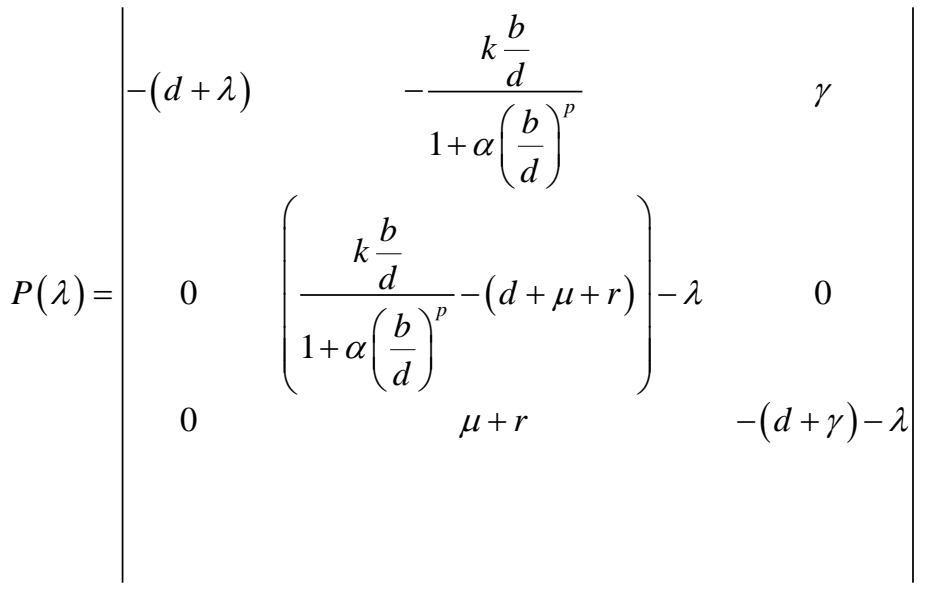

$$
\begin{aligned}
& -(d+\lambda)\left[\left(\frac{k(b / d)}{1+\alpha(b / d)^{p}}-(d+\mu+r+\lambda)\right)(-(d+\gamma+\lambda)]=0\right.
\end{aligned}
$$

Obviously, (3) has three roots $\lambda_{1}=-d, \lambda_{2}=\frac{k \frac{b}{d}}{1+\alpha\left(\frac{b}{d}\right)^{p}}-(d+\mu+r), \lambda_{3}=-(d+\gamma)$.

Let $R_{0}=\frac{k \frac{b}{d}}{(d+\mu+r)\left(1+\alpha\left(\frac{b}{d}\right)^{p}\right)}$

Hence, if $R_{0}<1$, then the disease free equilibrium point $E_{0}$ is locally asymptotically stable.

Proposition 2: $R_{0} \rightarrow 0$ as $b \rightarrow \infty$ for $\alpha>1$

\section{Proof:}

$$
\begin{aligned}
& R_{0}=\frac{k \frac{b}{d}}{(d+\mu+r)\left(1+\alpha\left(\frac{b}{d}\right)^{p}\right)} \\
& \frac{\mathrm{d} R_{0}}{\mathrm{~d} b}=\frac{\left[\frac{k}{d}(d+\mu)\left(1+\alpha\left(\frac{b}{d}\right)^{p}\right)\right]-\alpha\left(\frac{b}{d}\right)^{p-1} k \frac{b}{d}+(d+\mu+r)}{\left[(d+\mu+r)\left(1+\alpha\left(\frac{b}{d}\right)^{p}\right)\right]^{2}}
\end{aligned}
$$




$$
\begin{aligned}
& \text { i.e. } \frac{\mathrm{d} R_{0}}{\mathrm{~d} b}=\frac{\frac{k}{d}(d+\mu+r)}{(d+\mu+r)^{2}\left(1+\alpha\left(\frac{b}{d}\right)^{p}\right)^{2}} \\
& \frac{\mathrm{d} R_{0}}{\mathrm{~d} b}=\frac{k}{d(d+\mu+r)\left(1+\alpha(b / d)^{p}\right)^{2}}
\end{aligned}
$$

Hence, $\frac{\mathrm{d} R_{0}}{\mathrm{~d} b}>0, R_{0} \rightarrow 0$ as $b \rightarrow \infty$

$$
\lim _{b \rightarrow \infty} R_{0}=\frac{\lim \frac{k}{d}}{(d+\mu+r)\left(p \alpha\left(\frac{b}{d}\right)^{p-1}\right)}=0
$$

\section{Stability Analysis of the Endemic Equilibrium}

Let the endemic equilibrium $E_{*}=\left(S_{*}, I_{*}, R_{*}\right)$ where each component corresponds an earlier value.

Let

$$
x=S-S_{*}, \quad y=I-I_{*}, \quad z=R-R_{*}
$$

Then,

$$
\begin{aligned}
\frac{\mathrm{d} x}{\mathrm{~d} t} & =-d x-\frac{k I_{*} X}{1+\alpha S_{*}^{p}+\beta I_{*}^{q}}-\frac{k S_{*} y}{1+\alpha S_{*}^{p}+\beta I_{*}^{q}}+\gamma z+\text { non linear terms } \\
\frac{\mathrm{d} y}{\mathrm{~d} t} & =\frac{k I_{*} X}{1+\alpha S_{*}^{p}+\beta I_{*}^{q}}+\frac{k S_{*} y}{1+\alpha S_{*}^{p}+\beta I_{*}^{q}}-(d+\mu+r) y+\text { non linear terms } \\
\frac{\mathrm{d} z}{\mathrm{~d} t} & =(\mu+r) y-(d+\gamma) z
\end{aligned}
$$

Then

$$
\begin{aligned}
& \left(\begin{array}{l}
x^{1} \\
y^{1} \\
z^{1}
\end{array}\right)=A\left[\begin{array}{l}
x \\
y \\
z
\end{array}\right] \\
& \text { where } A=\left[\begin{array}{ccc}
-\left(d+\frac{k I_{*}}{1+\alpha S_{*}^{p}+\beta I_{*}^{q}}\right) & -\frac{k S_{*}}{1+\alpha S_{*}^{p}+\beta I_{*}^{q}} & \gamma \\
\frac{k I_{*}}{1+\alpha S_{*}^{p}+\beta I_{*}^{q}} & \left(\frac{k S_{*}}{1+\alpha S_{*}^{p}+\beta I_{*}^{q}}-(d+\mu+r)\right) & 0 \\
0 & (\mu+r) & -(d+\gamma)
\end{array}\right] \\
& P(\lambda)=\left|\begin{array}{ccc}
-\left(d+\frac{k I_{*}}{1+\alpha S_{*}^{p}+\beta I_{*}^{q}}+\lambda\right) & -\frac{k S_{*}}{1+\alpha S_{*}^{p}+\beta I_{*}^{q}} & \gamma \\
\frac{k I_{*}}{1+\alpha S_{*}^{p}+\beta I_{*}^{q}} & \left(\frac{k S_{*}}{1+\alpha S_{*}^{p}+\beta I_{*}^{q}}-(d+\mu+\gamma+\lambda)\right) & 0 \\
0 & (\mu+r) & -(d+\gamma+\lambda)
\end{array}\right|
\end{aligned}
$$


by solving, the characteristic equation $P(\lambda)$ is

$$
\lambda^{3}+a_{1} \lambda^{2}+a_{2} \lambda+a_{3}=0
$$

where

$$
\begin{aligned}
a_{1}= & 3 d+\gamma+\mu+r+\frac{k I_{*}}{1+\alpha S_{*}^{p}+\beta I_{*}^{q}}+\frac{k I_{*}}{1+\alpha S_{*}^{p}+\beta I_{*}^{q}}>0 \\
a_{2}= & 3 d^{2}+2 d \mu+2 d r+2 d \gamma+\gamma \mu+\gamma r+\frac{2 d k I_{*}}{1+\alpha S_{*}^{p}+\beta I_{*}^{q}}+\frac{\mu k I_{*}}{1+\alpha S_{*}^{p}+\beta I_{*}^{q}} \\
& +\frac{r k I_{*}}{1+\alpha S_{*}^{p}+\beta I_{*}^{q}}-\frac{d k S_{*}}{1+\alpha S_{*}^{p}+\beta I_{*}^{q}}-\frac{\gamma k S_{*}}{1+\alpha S_{*}^{p}+\beta I_{*}^{q}}>0 \\
a_{3}= & d^{3}+\gamma d^{2}+\gamma d \mu+\gamma d r-\mu d^{2}-r d^{2}-\frac{\gamma d k S_{*}}{1+\alpha S_{*}^{p}+\beta I_{*}^{q}}-\frac{\gamma \mu k I_{*}}{1+\alpha S_{*}^{p}+\beta I_{*}^{q}}-\frac{\gamma r k I_{*}}{1+\alpha S_{*}^{p}+\beta I_{*}^{q}}>0
\end{aligned}
$$

A straight forward calculation yields $a_{2} a_{1}-a_{3}>0$. Then, it follows from Routh-Hurwitz criteria that all characteristics roots have negative real parts. Thus, the endemic equilibrium is locally asymptotically stable.

\section{Numerical Simulations}

To see the dynamical behavior of system (2), we solve the system by Runge-Kutta Felhberg 45(RKF 45) method using the parameters; $\gamma=1.5, \mu=0.19, \rho=2, q=2, b=3.1, d=2.29, k=9, \alpha=3.1$ and $\beta=4.7$ with different values for the treatment term

a) In Figure 1, $r=0.1$.

b) In Figure 2, $r=0.4$.

c) In Figure 3, $r=0.7$.

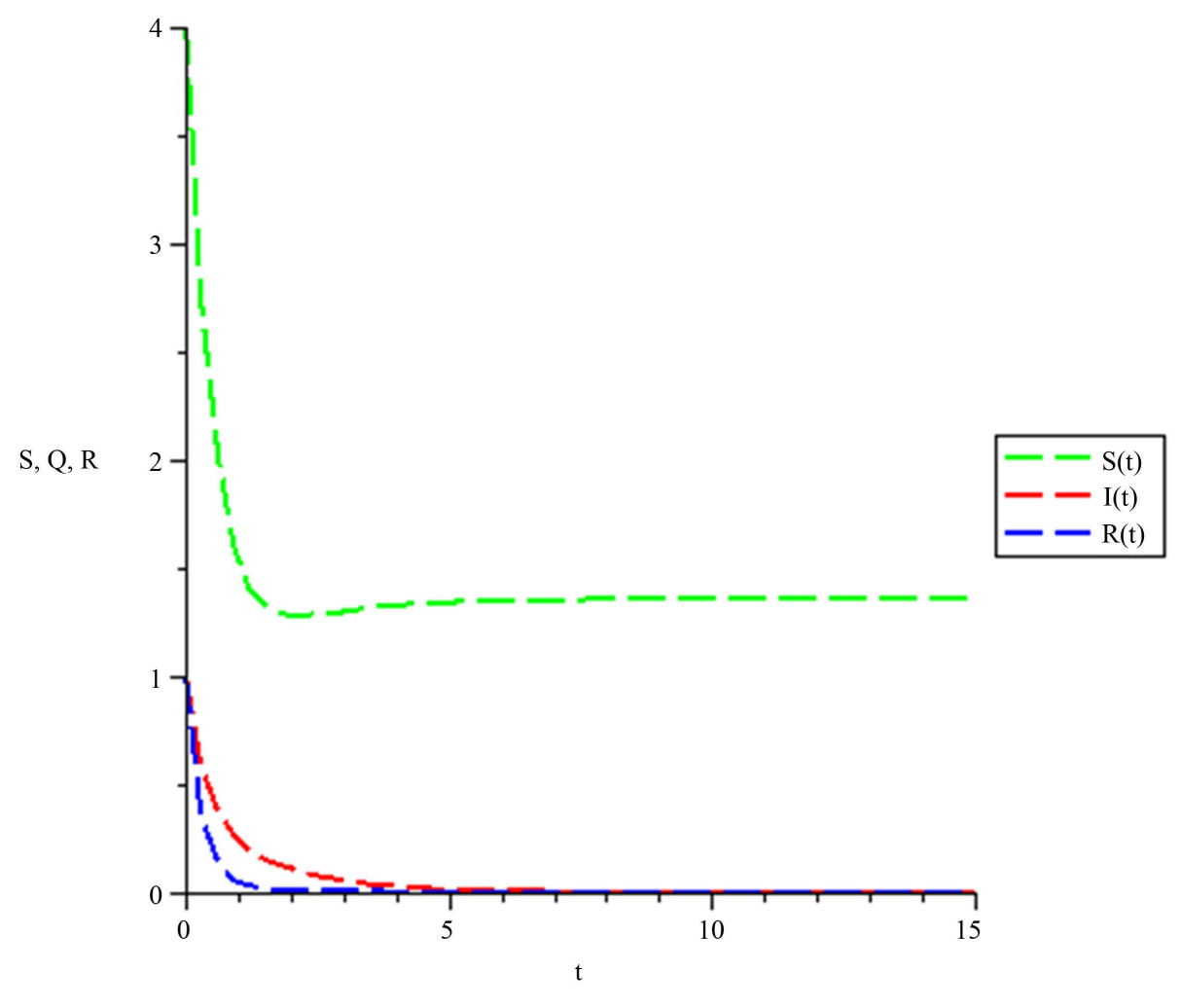

Figure 1. Graph of $S(t), I(t)$ and $R(t)$ when $\gamma=1.5, \mu=0.19, \rho=2, q=2, b=3.1, d=2.29, k=$ $9, \alpha=3.1$ and $\beta=4.7$. 


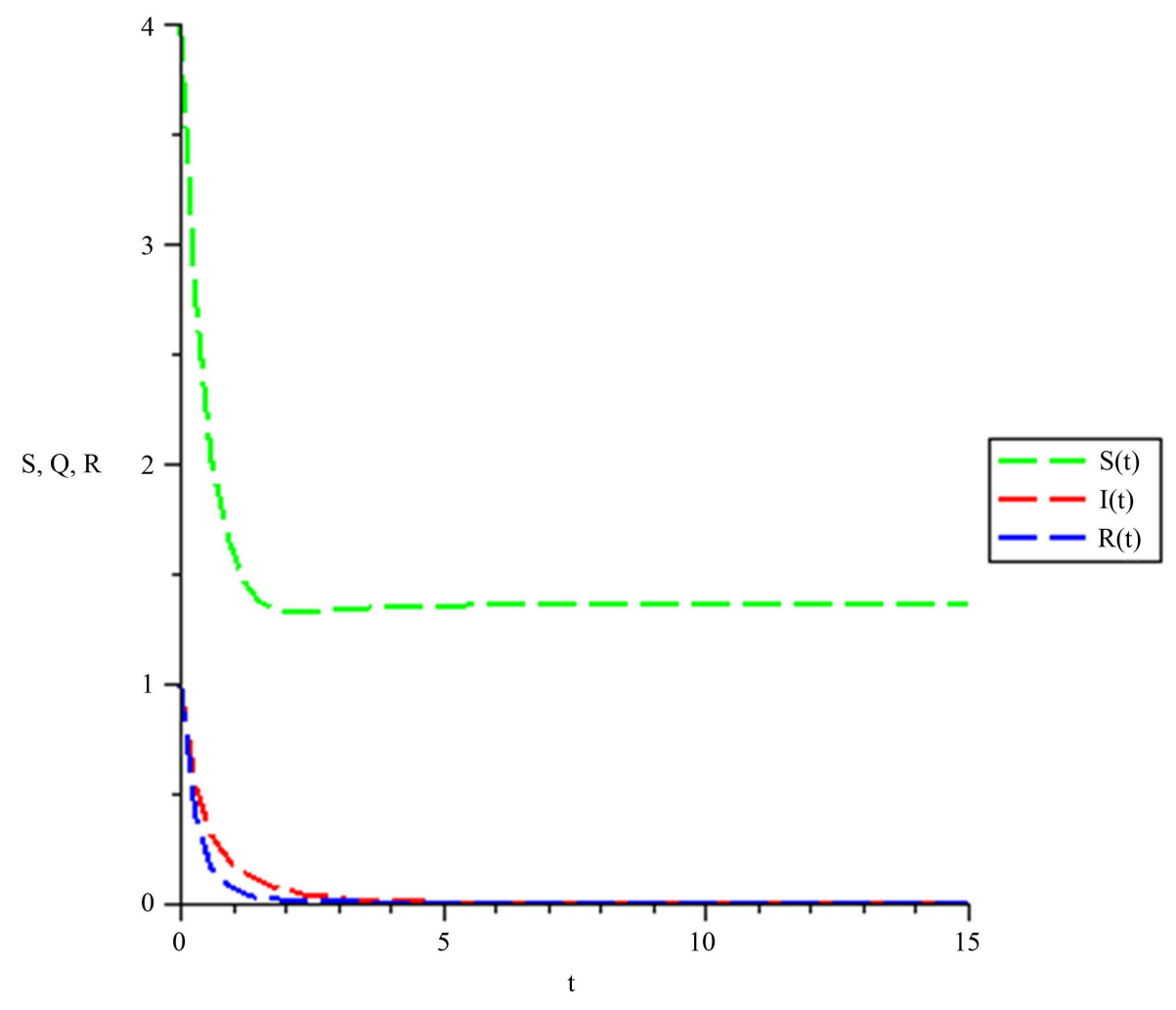

Figure 2. Graph of $S(t), I(t)$ and $R(t)$ when $\gamma=1.5, \mu=0.19, \rho=2, q=2, b=3.1, d=2.29, k=$ $9, \alpha=3.1$ and $\beta=4.7$.

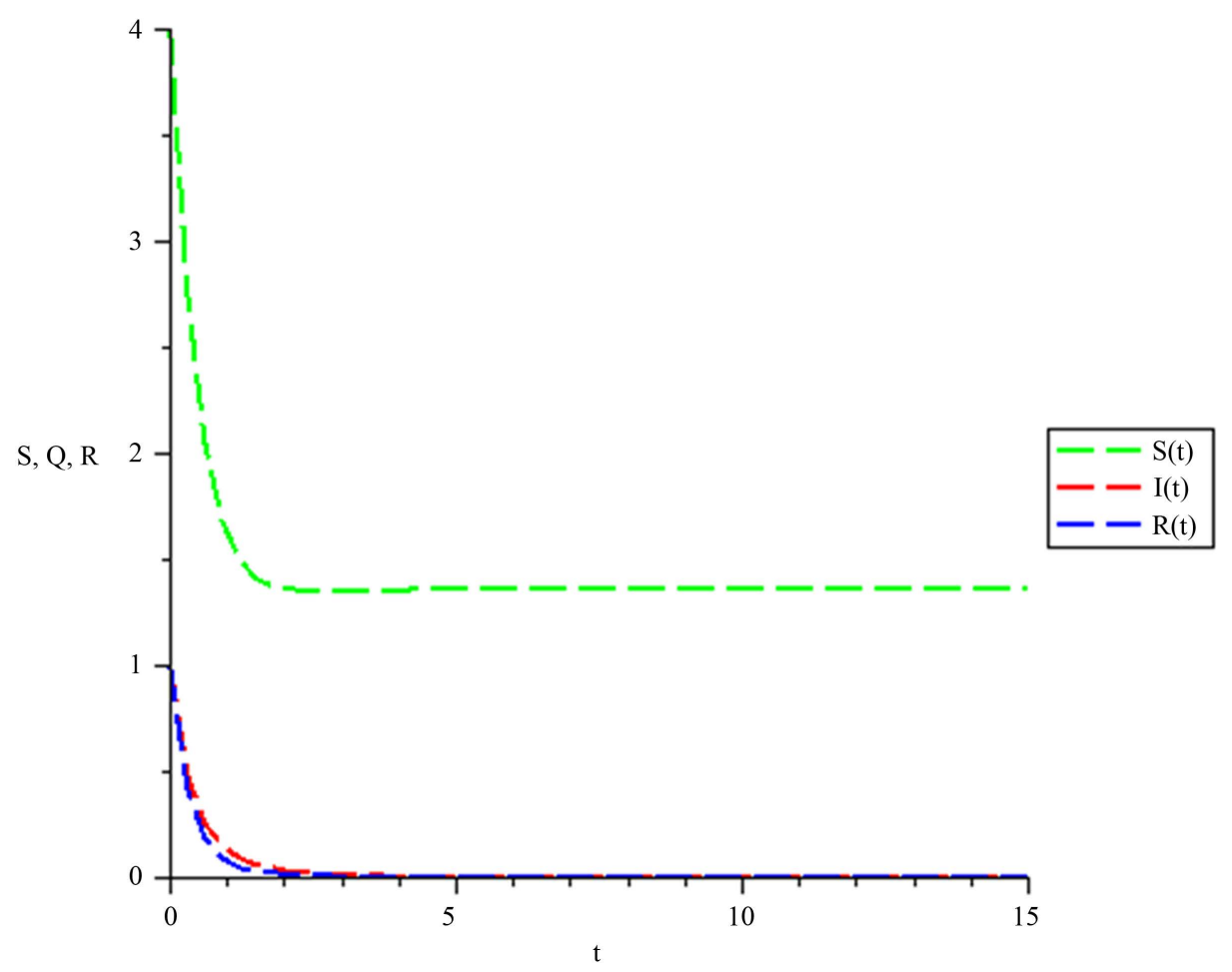

Figure 3. Graph of $S(t), I(t)$ and $R(t)$ when $\gamma=1.5, \mu=0.19, \rho=2, q=2, b=3.1, d=2.29, k=9$, $\alpha=3.1$ and $\beta=4.7$. 


\section{Conclusion}

In this paper, we have carried out the stability of the equilibrium states using some of the tested parameters from literature reviewed in this paper. The simulation is carried out using numerical software called "maple”. The effect of the treatment term in the model has been investigated and it shows that treatment has a positive effect on the endemic nature of the disease. The more the treatment is applied, the faster the disease fades out.

\section{References}

[1] Bernouilli, D. (1760) Essai d'une nouvelle analyse de la mortalite causse par la petite verole et des avantages de l'innoculation pour al prevenir. In Memoires de Mathematiques et de Physique. Academic Royale Des Science, Paris, $1-45$.

[2] Hethcote, H.W. (2000) The Mathematics of Infectious Disease. SIAM Review, 42, 599-653. http://dx.doi.org/10.1137/S0036144500371907

[3] Capasso, V. and Serio, G. (1978) A Generalization of the Kermack-Mckendrick Deterministic Epidemic Model. Mathematical Biosciences, 42, 41-61. http://dx.doi.org/10.1016/0025-5564(78)90006-8

[4] Ruan, S. and Wang, W. (2003) Dynamical Behavior of an Epidemic Model with a Nonlinear Incidence Rate. Journal of Differential Equations, 188, 135-163. http://dx.doi.org/10.1016/S0022-0396(02)00089-X

[5] van den Driessche, P. and Watmough, J. (2000) A Simple SIS Epidemic Model with a Backward Bifurcation. Journal of Mathematical Biology, 40, 525-540. http://dx.doi.org/10.1007/s002850000032

[6] Xiao, D.M. and Ruan, S.G. (2005) Global Analysis of an Epidemic Model with a Nonlinear Incidence Rate. Preprint.

[7] Jasmine, D.E.C. and Amirtharaj, H. (2014) A Modified SIR Epidemic Model with Immigration and Generalized Saturated Incidence Rate Function. International Journal of Science and Research, 3, 440-443.

[8] Chauchan, S., Misra O.P. and Dhar, J. (2014) Stability Analysis of SIR Model with Vaccination. American Journal of Computational and Applied Mathematics, 4, 17-23.

[9] Kaddar, A. (2010) Stability Analysis in a Delayed SIR Epidemic Model with a Saturated Incidence Rate. Nonlinear Analysis: Modelling and Control, 15, 299-306.

[10] Pathak, S., Maiti, A. and Samanta, G.P. (2010) Rich Dynamics of an SIR Epidemic Model. Nonlinear Analysis: Modelling and Control, 15, 71-81. 